\title{
Personalized Design and Implementation of IT Operation and Maintenance Monitoring Portal in Colleges and Universities
}

\author{
Gengsheng Deng ${ }^{1}$, Guiping Zhang ${ }^{2}$, Fu AiYing ${ }^{1}$, Weiping Zou ${ }^{1}$ \\ ${ }^{1}$ Network Center, Nanchang University, Nanchang, Jiangxi Province, China \\ ${ }^{2}$ Gongqing College, Nanchang University, Gongqing city, Jiangxi Province, China \\ dgsheng@ncu.edu.cn, karen-apple@163.com,ayfu@ncu.edu.cn, zwp@ncu.edu.cn
}

Keywords: IT operation and maintenance, personalized, monitoring platform, software design

\begin{abstract}
Due to the various types and a large amount of network software and hardware equipment in colleges and universities, as well as kinds of business systems are widely used, the brands involved are complicated. However, there is still not a special IT operation and maintenance system which is applicable for colleges and universities. Therefore, in order to meet the needs of the whole IT operation and maintenance monitoring, the operation and maintenance personnel need to log in the NMS, security management software and application system one by one to obtain the running status monitoring information of these applications or systems. In this paper, the author analyzed that by constructing the personalized application service monitoring portal system, so as to integrate all kinds of information collected into the portal for personalized display and the operation and maintenance personnel only need to log in the portal to monitor the information of their respective concerns, rather than logging in each separate system. As the information collected by the system is analyzed and processed, the technical requirements for the operation and maintenance monitoring personnel and daily monitoring workload are reduced, thus the work efficiency is greatly improved.
\end{abstract}

\section{INTRODUCTION}

With the expansion of the campus network size, as well as a large number of various application systems' construction, people's requirements of IT resource management refinement and service are getting higher and higher.IT operation and maintenance of colleges and universities is an important routine work, which has the characteristic of heavy workload, involving a wide range and high demand(Pengxian Lin, 2016).At present, colleges and universities deploy network management software, network security equipment, authentication and billing system, mail system, virtualization services and other types of applications and platforms to support the school's information services. In order to achieve the daily operation and maintenance, we need to get the information of current status and statistics of various systems, applications, hardware and software in real time. A system which can include all large and complete IT operation and maintenance management may be an ideal solution for IT resources and application systems to achieve unified monitoring, but because the recent operation and maintenance management system has its own advantages and disadvantages, they can not meet the needs of the entire school to achieve IT operation and maintenance monitoring, especially for the application system (Huiyun $\mathrm{Wu}, 2015$ ).

In this paper we designed an easy-to-extend, personalized operation and maintenance monitoring portal system which can collectively display the status and statistics collected from each system and displays the content of interest according to the type and preferences of the user, thus it will greatly improve the efficiency of access to monitoring information. At the same time the system can be targeted through the set of some rules in the system to display alarm information.

\section{PROBLEM ANALYSIS}

\subsection{Analysis of the status quo and the existing problems}

As the recent University IT environment has the following characteristics: there are a wide variety of types and quantities of software and hardware equipment, complex network environment, various types of business systems are widely used, involving complex manufacturers and brands etc, so there is not a particularly applicable IT operation and maintenance system to colleges and universities (Yanzhao Lu, 2015). Our school has deployed some operation and management software, such as network repair platform, authentication and billing system, wireless network management software, Cacti network monitoring, dynamic ring monitoring system, network auditing, site statistics, log systems, etc., combined with some application systems and network security equipment and the system comes with the management platform to support the school's operation and maintenance management and monitoring work.The daily operation status monitoring is an important work in the daily operation and maintenance process, but the current monitoring methods are scattered and the monitoring system is isolated from each other. We need to enter the system to view the data, so that the work efficiency is low. Because the network management and application system information volume range is relatively large, the data that the manager is concerned is not intuitive, therefore, technical capacity requirements for the staff are relatively high. Because we need to extract the 
running data from each system and application system when we issue the operational report, the monitoring personnel need to remember a large number of user name password to access the system, we need to invest more technical human resources. In addition, it requires a higher authority for the monitoring staff to view some system running data account and it is not conducive to the system security management.

\subsection{Problems to be solved}

In order to solve the problems mentioned above, we need a unified information / data integration platform to integrate all the information which is about the individual network management systems within the campus and all kinds of operation and maintenance management of the information concerned in a unified monitoring platform. In a unified platform, all staff can deal with daily monitoring and management work through the use of single sign-on and identity authentication in accordance with their respective scope of authority. The construction of a unified monitoring platform needs to address the following four issues:

(1). Information acquisition: According to the importance of information content and the update situation, we can use real-time access and timing access to obtain information. The contents of the information include the deployment of network management software, application systems and other operating status and statistical data, such as hardware and software equipment CPU, memory and other basic information, service response time, load, throughput, application system access and use,etc(Hua Zhang,2014).There are also some information on the Internet that needs attention, such as anti-spam organization related blacklist, website or system vulnerability information list. The diversity of information sources requires the system to be able to perform data acquisition in real time or on a regular basis in a variety of heterogeneous ways.

(2). Information processing: We need to format the original information with different contents and formats we obtained, analyze the data base on the content that needs to be monitored, and form the corresponding monitoring indicators before the next step, such as storage, display or alarm notification. So the system needs to be able to flexibly process and store a variety of data formats.

(3). Information display: The information obtained by the system needs to be displayed centrally. On the one hand, as the information obtained by the system are in a large number and many kinds, with the increase in the number of devices and systems, the need to display the contents of the portal will be more and more. The types displayed in the page are not the same, some are suitable for the chart display, while some for the list display. On the other hand, the display of information requires both PC and mobile access, at the same time different management staff are concerned about different content. Network administrators may be more concerned about the information of network running status , security administrators are more concerned about security-related information, so the display page needs to support personalized customizable.

(4). Notice Alert: A stable and reliable monitoring system should be able to promptly inform the appropriate staff prompt, alarm, error, etc. There will be a variety of errors and warning in a system at any time, so the management program needs to be able to react quickly and deal with all kinds of errors instantly. The alarm error message includes the alarm error information contained in the information content generated and obtained by the system itself.

\section{DESIGN AND IMPLEMENTATION OF IT OPERATION AND MAINTENANCE PORTAL IN COLLEGES AND UNIVERSITIES}

\subsection{Design principles and architecture design}

In order to implement the system better, this paper puts forward the following design principles according to the demand: practical principle, expansibility principle, stability principle and maintainability principle.

(1). Practical principle: The system is focused on monitoring and display. The system itself does not replace the existing IT operation and maintenance management system to achieve large and complete monitoring and management functions, but use the most suitable tools or applications to achieve the most suitable function. The system then display the information collected from it intensively, such as collecting hardware or server running status and legend from Cacti.

(2). Expansibility principle: As the system needs to obtain all kinds of structures and different types of data information from a wide range of hardware and software equipment, the methods required are also varied. The system should have a better scalability in order to deal with the various types of hardware and software equipment. The same is for the displayed information in different content and manner.

(3). Stability principle: The system should be designed to reduce the coupling between the various applications and services, to avoid the failure of a single service to affect the normal operation of the entire system. The system should provide a flexible deployment model, data collection and WEB should be able to achieve balanced load and distributed deployment.

(4). Maintainability principle: The system needs to collect information from various heterogeneous hardware and software devices so it should have a good collection and monitoring capability. The system can also manage the information intensively and quickly locate the possible point of failure.

The overall architecture of the monitoring portal can be logically divided into four levels: acquisition layer, data layer, application layer and presentation layer.

The collection layer mainly collects the data of the target object or system according to the demand, the performance, the availability, the statistical information and so on, realizes the information acquisition and the preliminary processing to the original information, and then initially clean and process the original information collected. The data layer is used to further process the information of the initial processing of the mining layer, complete the data formatting and provide a unified data 
interface, finally to achieve the function of unified storage and management for status, performance, availability, statistics and other information .Application layer is to provide a sound management function modules based on the data layer, including template management, view management, timer management, plug-in management, resource management, fault handling, alarm management module. Performance layer, all application functions are unified, providing a graphical management interface based on the Web, a unified user authentication and login interface, the user can customize the interface to customize the personalized user interface.

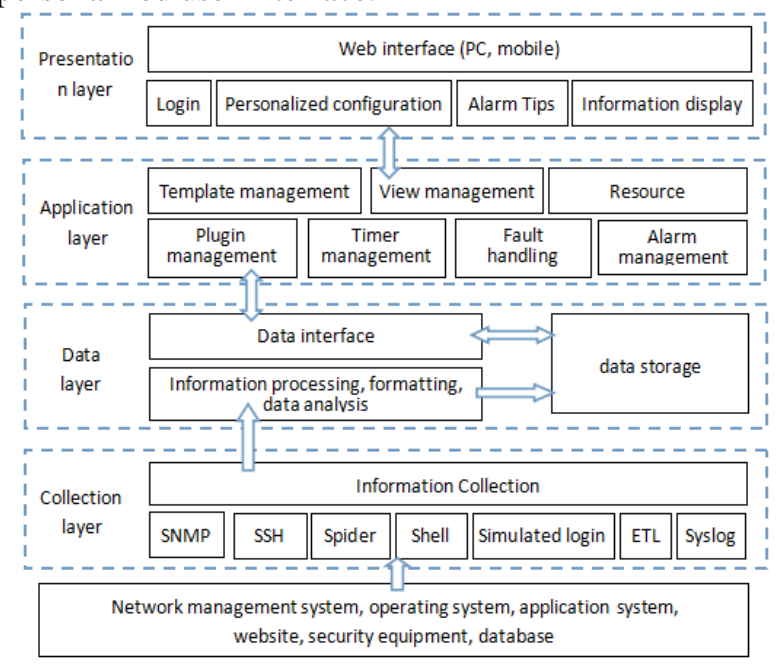

Figure 1: Schematic diagram of the overall system architecture.

\subsection{Functional design}

System functions include user management, template management, view management, timer management, plug-in management, resource management, fault handling, alarm management and other functional modules.

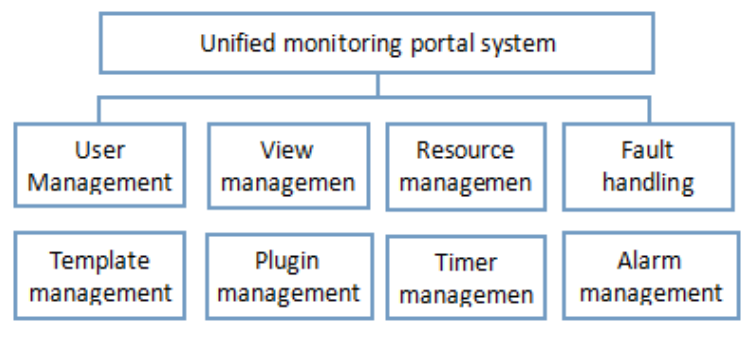

Figure 2: Schematic diagram of the functional module.

In order to achieve better scalability, the system uses plug-ins to manage a variety of information collection, storage and alarm processes and rules, through developing multiple types of plugins by customizing, combined with SNMP, SSH, web crawler, simulated login, database extraction and other ways to actively acquire the data of a variety of network equipment, servers, application system middleware and private business system .It can also use Syslog, SNMP TRAP way to obtain the relevant information passively. Each plug-in is independent from each other. A plug-in need to implement data acquisition, data storage initialization, data interface API, etc. If the displayed content is more complex, you can define the display view in the plug-in.
The system needs to manage and issue tasks uniformly, the collection plug-in can be distributed in different machines for information collection, it can access and modify data through the standard data interface

The administrator set the frequency of the collection task, the system will automatically take the initiative to polling the collected resources. All the collected data will be stored in the database after formatting. This process does not require any administrator intervention and operation. The following steps are usually taken to handle the various information collected form the target object:1, the information pre-processing, to remove some invalid data; 2, complete the data format; 3 , store the formatted data; 4, initial threshold comparison;5, issue an alarm for the information which is not within the threshold range, and use the pop-up window, voice, mail, instant messaging, etc. to inform the user whom had been subscribed. Administrators can view IT business status from time to time through a browser and get data analysis and reporting. When the administrators are off work or offline, they can also receive the alarm information sent by the monitoring system.

\subsection{Personalized design}

The personalized design of the system mainly includes three aspects: access control, content customization and appearance setting.

(1). Access control: Different types of users have different access to the recources in the system, the content they can customize is also different. The system has access control of the resources through the user, the role and the authority. The user must explicitly belong to a role. Each role has the corresponding access right, and the system administrator has the access right to increase or decrease the access authority of the role. Only by specifying the user and authority can the system better provide personalized service (Weiwan Liu, 2016).

(2). Content customization. The user can set what content can be displayed within the scope of their own authority and what content to be shielded; users can subscribe to the alarm information and set the content, methods, levels and so on of the notification.

(3). Appearance settings. Users can choose page layout, style and color, etc. according to their preferences and re-adjust the appearance in accordance with their personal preferences.

\section{SYSTEM IMPLEMENTATION AND EFFECT}

The system uses an open source LNMP environment and combine Memcached to optimize data query. Among them, all kinds of data interfaces are implemented in Restful style API. The interface response adopts the JSON format by default, so as to achieve the data unification between the PC terminal and the mobile terminal. The WEB interface of the presentation layer is implemented with the Extjs framework. The system can be deployed on most servers and you can specify one of them as the primary server. The acquisition task can be assigned to different servers, each timer on the server triggers each collection task, and the acquired information is saved to the main server 
Information gathering is an important part of the system that needs to be implemented. Because there are various kinds of objects, the methods needed are different. At present, the following strategies are adopted to write the corresponding plug-ins to realize the collection of heterogeneous object information:

(1). If the target object provides the API interface to obtain data, the API interface is called first to obtain it.

(2). If the target system uses standard SNMP, WMI and SYSLOG, the data can be integrated into the network management software and log server system. The system obtains data from the network management software log server, if it can not be integrated into the network management software, and then it will call the protocol to obtain data.

(3). With no user login sites and systems, Crawl can be used before format.

(4). With users login sites and systems, you can use analog login and then crawl;

(5). Extract database information. In this situation we need to analyze the database structure, the principle is that you can not move the database if it is not necessary.

(6). For a small number of information which can not be collected actively, we can write the script PUT to the corresponding data interface.

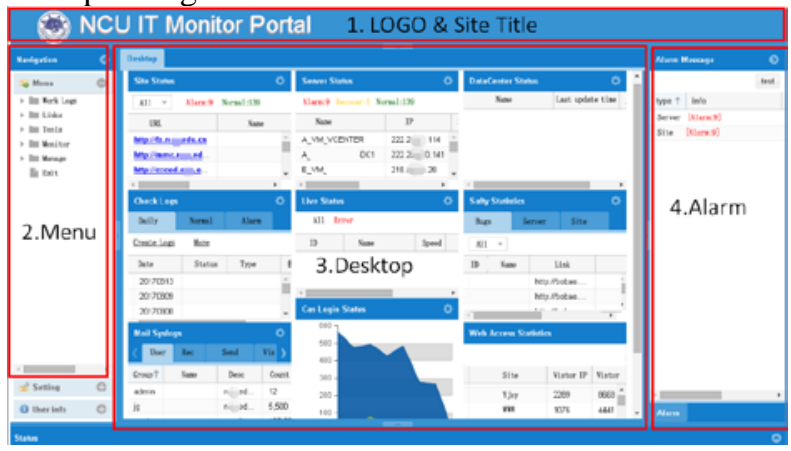

Figure 3: Screenshot of application service monitoring system.

The main monitoring services in my school are server status, website status, host security information, website security information, various application states, virtualization platforms and so on. At present, the server status, site status, host security information, site security information, part of the application status and other types of information content have been integrated into the portal system.

The main source of information includes: Cacti network management software, mail system, unified identity authentication, data exchange platform, 360 security guards Enterprise Edition, Piwiki website access statistics, vulnerability platform, Webscan website security and other systems or content. Through this integration, the work efficiency of the daily monitoring staff has been greatly improved:

(1) No longer for the user to remember more than one system user name and password, just remember the portal password.

(2) No longer for the user to log on each system or website to view the retrieval data, and the data and indicators of each system can be displayed on the front page of the monitoring portal; take security information for example, in the past we had to pay attention to the internal firewall, security guards, Webscan and the major vulnerabilities, platforms, search engines and so on.

(3) The collected information has been processed and analyzed again, which reduces the technical requirements of the operation and maintenance personnel.

(4) It has changed the messages and alarms of different systems in the past, but to manage and send them in a unified way. Subscriptions such as page popups, mail messages and PIN messages have been implemented at present.

The system combines the inspection log and the small function of processing records in the portal, collect and sort out the common problems in the operation and maintenance process, and gradually form standard solutions;6. It has strengthened the cooperation ability between maintenance personnel.

\section{CONCLUSIONS}

Based on the actual situation of our university, this paper introduces the case of monitoring the portal system through building personalized application services After collecting the information of network management system, security management software and application system, they are processed and integrated into the portal system. Users can customize the content and interface they are interested in. They only need to log in to monitor the portal and the application service status without having to $\log$ in to each system to view or retrieve information, the system analyzes and processes the information collected by the system, reduces the technical requirements of the operation and maintenance monitoring personnel, reduces the workload of the operation and maintenance monitoring personnel, thereby greatly improves the work efficiency. We need to integrate more systems and applications in the future. At present, the information collected by each plug-in is more independent, so it is necessary to improve the quality and effect of monitoring in order to make a further analysis of the correlation between all kinds of information obtained.

\section{REFERENCES}

[1] Hua Zhang, 2014. Design of a lightweight monitoring architecture, Computer Applications and Software. 31(7), pp.86-88.

[2] Huiyun Wu, 2015. Development of IT operation and maintenance in universities at home and abroad. China education network. (7), pp.34-36.

[3] Pengxian Lin, 2016.Research and application of performance monitoring technology for low price Cloud Application, Zhejiang University.

[4] Weiwan Liu, 2016. Role based personalized portal design and implementation. wireless internet science and technology. (17), pp.63-64.

[5] Yanzhao Lu, 2015.Design and implementation of Information Center IT operation and maintenance monitoring system, University of Chinese Academy of Sciences. 